\title{
Diagnostic Modality for Evaluation of Right Ventricle in Chronic Thromboembolic Pulmonary Hypertension Patients
}

\author{
Mustafa Oguz, Tarik Kivrak', Murat Sunbul' ${ }^{2}$ Fuad Dede ${ }^{3}$, Bedrettin Yildizeli ${ }^{4}$, Bulent Mutlu ${ }^{2}$ \\ ORCID: \\ Mustafa Oguz: https://orcid.org/0000-0002-5165-1212 \\ Tarik Kivrak: https://orcid.org/0000-0002-5257-4810 \\ Murat Sunbul: https://orcid.org/0000-0002-7755-3370 \\ Bedrettin Yıldızeli: https://orcid.org/0000-0002-1316-4552 \\ Bulent Mutlu: https://orcid.org/0000-0003-0973-3422
}

Department of Cardiology, Van State Hospital, Van, ${ }^{1}$ Department of Cardiology, Firat University Hospital, Elazığ, ${ }^{2}$ Department of Cardiology, Marmara University Hospital, Istanbul, ${ }^{3}$ Department of Nuclear Medicine, Marmara University Hospital, Istanbul, ${ }^{4}$ Department of Thoracic Surgery, Marmara University Hospital, Istanbul, Turkey

\section{Abstract}

Background: Chronic thromboembolic pulmonary hypertension (CTEPH) is a progressive disease of pulmonary hypertension (PH) as a consequence obstructive of pulmonary arteries with thromboembolism. Augmented pulmonary vascular resistance and pulmonary artery pressure (PAP) can ultimately lead to the right ventricular dysfunction, which leads to adaptive and maladaptive changes. Right ventricle (RV) remodeling can cause clinical deterioration and RV failure. Aims and Objectives: We aimed to evaluate the RV functions with a new diagnostic modality 2-deoxy-2-(18F)-fluoro-D-glucose (FDG)-positron emission tomography (PET) in patients with CTEPH. Materials and Methods: We included 53 patients diagnosed with CTEPH who were planned to have pulmonary thromboendarterectomy (PEA) and did not have any contraindication for cardiac PET/computed tomography (CT). We performed transthoracic echo, cardiac PET/CT, 6-minute walk distance (6-MWT), and right heart catheterization 1 week before PEA surgery. Results: The patients divided into two groups according to RV/left ventricle (LV) FDG-PET uptake ratio, RV/LV $\leq 1$, and RV/LV $>1$. Six-MWD was significantly higher in the RV/LV $\leq 1$ group $(P=0.005)$. Pro-BNP was considerably higher in the $\mathrm{RV} / \mathrm{LV}>1$ group $(P=0.041)$. Conclusion: The present study aims to demonstrate the RV/LV FDG-PET uptake ratio could be used in the noninvasive diagnostic method in diagnosing, treatment strategy, and clinical follow-up in patients with CTEPH.

Keywords: Cardiac fluoro-D-glucose positron emission tomography, chronic thromboembolic pulmonary hypertension, pulmonary vascular resistance, right ventricular remodeling

\section{INTRODUCTION}

Chronic thromboembolic pulmonary hypertension is the development of progressive pulmonary hypertension $(\mathrm{PH})$ due to occlusion of pulmonary vascular bed and formation of structural changes by recurrent and organized pulmonary thromboembolism or in situ (in place) thrombosis. ${ }^{[1]}$ This causes increased pulmonary vascular resistance (PVR) and pulmonary arterial pressure in pulmonary vascular bed over time. Adaptive remodeling such as compensatory right ventricle hypertrophy and increased contractility occur

Received: 25-Jul-19 Revision: 18-Aug-19 Accepted: 01-Sep-19

Published Online: 25-Nov-19

\begin{tabular}{|l|l|}
\hline \multicolumn{2}{|c|}{ Access this article online } \\
\hline Quick Response Code: & Website: \\
\hline & \\
&
\end{tabular}

against increased afterload in RV. In time, these compensatory mechanisms become inadequate, and functional deterioration progresses by maladaptive changes in RV.

As a consequence of increased RV afterload, a clinical syndrome called RV failure develops due to insufficient blood flow in pulmonary vascular bed during resting or exercise and increased systemic venous pressure. ${ }^{[2]}$ This anatomical, physiological, and

Address for correspondence: Dr. Tarik Kivrak Assistant Professor, Department of Cardiology, Firat University Hospital, Elazığ, Turkey. E-mail: tarikkivrak@gmail.com

This is an open access journal, and articles are distributed under the terms of the Creative Commons Attribution-NonCommercial-ShareAlike 4.0 License, which allows others to remix, tweak, and build upon the work non-commercially, as long as appropriate credit is given and the new creations are licensed under the identical terms.

For reprints contact: reprints@medknow.com

How to cite this article: Oguz M, Kivrak T, Sunbul M, Dede F, Yildizeli B, Mutlu B. Diagnostic modality for evaluation of right ventricle in chronic thromboembolic pulmonary hypertension patients. Int J Cardiovasc Acad 2019;5:152-8. 
metabolic remodeling in RV is responsible for essential subclinical and clinical features of CTEPH, and RV functions determine mortality and morbidity. For these reasons, the development of novel diagnostic and therapeutic approaches to RV functions is gaining importance. In the analyses about $\mathrm{PH}$ in recent years, pulmonary vascular structure and right heart were analyzed as a combined group as a cardiopulmonary unit. ${ }^{[3]}$ Three primary mechanisms may be cause adaptive and maladaptive changes resulting in RV failure. These are ischemia due to pulmonary arterial hypertension (PAH), mitochondrial remodeling, and metabolic shift. ${ }^{[4]}$ The myocardial metabolism that changes from oxidative glycolysis to nonoxidative glycolysis in RV due to PAH can be used as a novel diagnostic modality for 2-deoxy-2-( $\left.{ }^{18} \mathrm{~F}\right)$ fluoro-D-glucose-positron emission tomography/computed tomography (FDG-PET/CT) to evaluate RV functions. In our study, we aimed to assess the relationship between RV functions in CTEPH patients with a diagnostic modality, FDG-PET/CT, and functional capacity.

\section{Methods}

This study included patients with CTEPH between June 2013 and January 2015 and planned to have pulmonary thromboendarterectomy procedure at our center. The Ethical Committee of Marmara University Medical School evaluated our study, and no objections were found for conduction. Informed consent forms were obtained from patients who found to be eligible for the survey regarding their approval for participation. It was a prospective study. A total of 53 patients with an exact diagnosis of CTEPH according to a multidisciplinary assessment at our center, who had no contraindication for a cardiac PET examination (overweight patients, pregnant, etc.). Patients with moderate-to-severe valve diseases, left heart failure with any reason, chronic obstructive lung disease with FEV1 below 50\%, chronic renal failure, diabetes mellitus, uncontrolled hypertension, coronary artery disease, and hypertrophic cardiomyopathy, and those that not approved to participate were excluded from the study.

Each patient had diagnostic workups 1 week before the operation. The same operator conducted all echocardiographic evaluations according to the recommendations of the American Society of Echocardiography guidelines ${ }^{[5]}$ using a Vivid 7 Ultrasound System (General Electric, Horten, Norway).
Right heart catheterization (RHC) was performed by the femoral approach using a Swan-Ganz catheter to confirm the diagnosis of PH. Cardiac output was calculated by the Fick method. Each patient presented the (6-MWD) according to the 6-MWD Protocol of American Thorax Surgery Society. ${ }^{[6]}$ The Qanadli Score is calculated in acute pulmonary thromboembolism by pulmonary CT angiography and pulmonary angiography and based on thrombus load in the pulmonary arterial bed. ${ }^{[7]}$ For the first time in this study, we use Qanadli score to calculated based on thrombus burden in pulmonary arterial bed in CTEPH patients. An integrated PET/CT system (Discovery-16 LS, GE Healthcare, USA) was used for imaging. It included include the entire free walls of the right and the (LV). Our center is used to 0.8 ratios as usual in right ventricle/LV ratio. ${ }^{[8,9]}$ Data computing and assessment were performed on a workstation (Advantage Windows Workstation 4.5, GE Healthcare, USA) following the imaging. The volume of interest was drawn over the right and LV free walls, and myocardial glucose involvement levels on these walls were obtained by measuring semiquantitative maximum standardized uptake values (SUV). Right/LV glucose metabolism proportions were calculated by calculating right and LV maximum SUV levels [Figure 1a-c].

\section{Statistical analysis}

SPSS 20 software (SPSS 20.0 for Windows, SPSS Inc., Chicago, IL, USA) was used for the statistical analyses of the study. The one-sample Kolmogorov-Smirnov test evaluated the distribution of data. If numerical data were normally distributed, then presented as mean \pm standard deviation; and if not normally distributed, then given as median (minimum-maximum). Chi-square test evaluated categorical variables. Customarily distributed numerical data were compared with Student's $t$-test, and nonnormally distributed statistical data was compared with the Mann-Whitney U-test. Pearson or Spearman's correlation tests were used for correlation analysis. The Pearson correlation was used in the linear relationship between two continuous variables. The Spearman correlation was used the monotonic relationship between two continuous or ordinal variables. $P<0.05$ values were considered as statistically significant.

\section{RESULTS}

A total of 53 patients diagnosed with CTEPH by a multidisciplinary evaluation, and those planned to have PEA
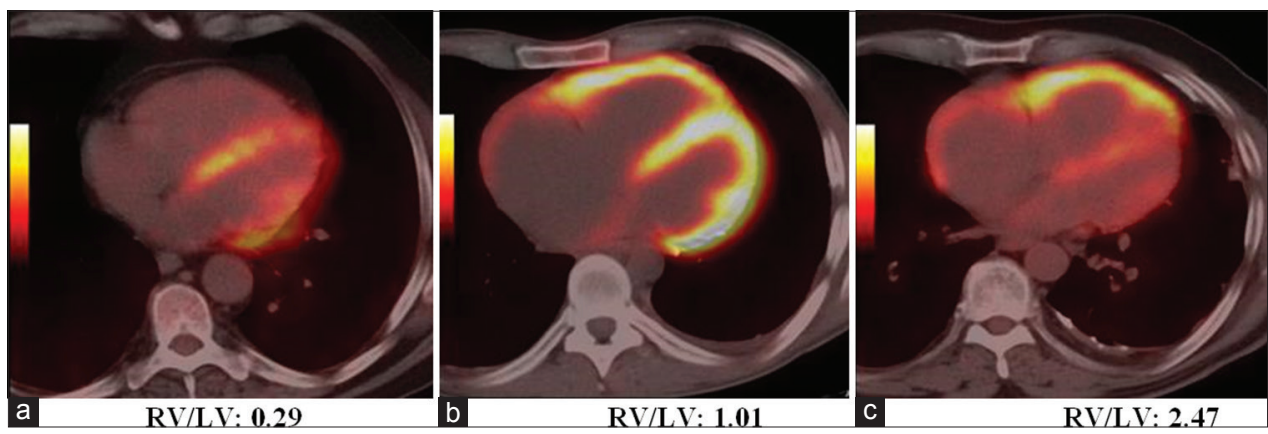

Figure 1: Sample of FDG PET uptake (a, b and $c)$ 
were included in the study. Mean age of the patients was $55 \pm 13.8$ years. Thirty-four patients were female $(64.2 \%)$ and 19 were male $(35.8 \%)$. General clinical characteristics and

\begin{tabular}{|c|c|}
\hline $\begin{array}{l}\text { Demographic and } \\
\text { clinical datas }\end{array}$ & Means ( $n: 53)$ \\
\hline \multicolumn{2}{|l|}{ Blood pressure (mmHg) } \\
\hline Systolic & $120.0(175-98)$ \\
\hline Mean & $92.8 \pm 17.4$ \\
\hline Diastolic & $75.4 \pm 11.1$ \\
\hline BMI & $27.8(19.5-43.7)$ \\
\hline 6-MWT (meters) & $266.5 \pm 161.7$ \\
\hline Pace (beat/min) & $86.6 \pm 14.1$ \\
\hline Qanadli score & $23.2 \pm 8.1$ \\
\hline \multicolumn{2}{|l|}{ Symptom } \\
\hline Present & $43(\% 81.1)$ \\
\hline None & $10(\% 18.9)$ \\
\hline $\mathrm{WBC},\left(\times 10^{3} / \mu \mathrm{L}\right)$ & $7.6(2.8-18.9)$ \\
\hline Hemoglobin, (g/dL) & $13.0 \pm 2.7$ \\
\hline Hematocrit, $(\%)$ & $38.8 \pm 6.1$ \\
\hline Thrombocyte, $\left(\times 10^{3} / \mu \mathrm{L}\right)$ & $233.5(83-690)$ \\
\hline Creatinine (mg/dL) & $0.86 \pm 0.29$ \\
\hline BUN (mg/dL) & $19.0 \pm 9.2$ \\
\hline D-dimer (mg/L) & $0.4 \pm(0.09-2.87)$ \\
\hline Uric acid (mg/dL) & $7.6 \pm 2.6$ \\
\hline ProBNP (pg/ml) & $986(12-17020)$ \\
\hline $\mathrm{CRP}(\mathrm{mg} / \mathrm{L})$ & $8.4(0.9-75.0)$ \\
\hline
\end{tabular}

BMI: Body mass index, 6-MWT: 6 Minute Walk Test, CRP: C reactive protein, WBC: White blood cell

Table 2: Echocardiography and right heart catheterization parameters of study population

\begin{tabular}{lc}
\hline ECHO and RHC Parameters & Means $(\boldsymbol{n}: 53)$ \\
\hline TAPSE (mm) & $14.4 \pm 4.3$ \\
MPI (mm) & $0.63 \pm 0.17$ \\
RVS (mm) & $10.7 \pm 3.1$ \\
RA area $\left(\mathrm{cm}^{2}\right) / \mathrm{LA}$ area $\left(\mathrm{cm}^{2}\right)$ & $23.9 \pm 7.7 / 16.1 \pm 4.3$ \\
RVEDD (mm) & $40.1 \pm 6.7$ \\
LVEDD (mm)/LVESD (mm) & $43.5 \pm 6.0 / 27.7 \pm 4.9$ \\
sPAP (mmHg) & $71.5 \pm 28.4$ \\
Systolic Pulmonary Artery Pressure (mmHg) & $76.5 \pm 27.3$ \\
Mean Pulmonary Artery Pressure (mmHg) & $48.1 \pm 15.6$ \\
Diastolic Pulmonary Artery Pressure (mmHg) & $29.2 \pm 11.9$ \\
Wedge (mmHg) & $10.9 \pm 4.2$ \\
Cardiac Output (lt/dk) & $4.2 \pm 1.7$ \\
Cardiac Index (lt/dk/m²) & $2.3 \pm 1.0$ \\
Transpulmonary gradient (mmHg) & $38.0 \pm 15.6$ \\
Diastolic pulmonary gradient (mmHg) & $18.5 \pm 10.9$ \\
PVR (woods)/SVR (woods) & $10.4 \pm 6.3 / 21.8 \pm 9.0$ \\
\hline RVEDD: Right ventricle end-diastolic diameter LVEDD: Left ventricle \\
end-diastolic diameter; LVESD: left ventricle end systolic diameter; \\
EF: Ejection fraction; RA: Right atrium area; LA: Left atrium area; \\
sPAP: Systolic pulmonary arterial pressure; TAPSE: tricuspid annular \\
plane systolic excursion; RVS: tricuspid annulus tissue Doppler systolic \\
velocity; LV: Left ventricle
\end{tabular}

basal laboratory values of the patients are shown in Table 1 . We have no data on their clinical symptoms and disease's duration.

Decreases in RV systolic function parameters and increases incorrect heart diameters were observed before the operation in patients. PAP, PVR, and the transpulmonary gradient were found to be high, and cardiac output and cardiac index were found to be low in RHC [Table 2].

The patient population was separated into two groups according to right ventricle/LVFDG-PET uptake ratio. Groups formed according to the right ventricle uptake less than $L V$ Group $1(\mathrm{RV} / \mathrm{LV} \leq 1)$ and proper ventricle uptake equals to and more than LV Group $2(\mathrm{RV} /$ LV >1). Twenty-seven patients were Group 1 and 25 patients were Group 2. Six-MWD, Qanadli score, Pro-BNP, Echo parameters, and RHC findings were compared between groups [Table 3]. Six-MWD was high in Group 1. The Qanadli score was calculated to be lower in Group 1, but it was not statistically significant. Pro-BNP was high in Group 1 when compared with Group 2. There were no significant differences between groups regarding echocardiography and RHC parameters [Table 3].

Correlation analyses were performed to determine the associations between clinical findings and RV/LV FDG-PET uptake levels in patients. A negative correlation was established between 6-MWD and RV/LV FDG-PET uptake ratio [Figure 2]. Furthermore, a positive relationship was found between increased Qanadli score, which determines the thrombus burden in the pulmonary arterial bed and RV/LV FDG-PET uptake ratio. There was a positive correlation between laboratory parameters of D-dimer and Pro-BNP and RV/LV FDG-PET uptake. There were no correlations between echocardiographic parameters of tricuspid annular plane systolic excursion (TAPSE), Myocardial Performance Index, and RV tissue systolic Doppler wave, which are frequently used to evaluate RV functions, and RV/LV FDG-PET uptake. There was a significant correlation between echocardiographic parameters of right ventricle area, LV diameter, and systolic pulmonary arterial pressure and RV/LV FDG-PET uptake [Figure 3]. There

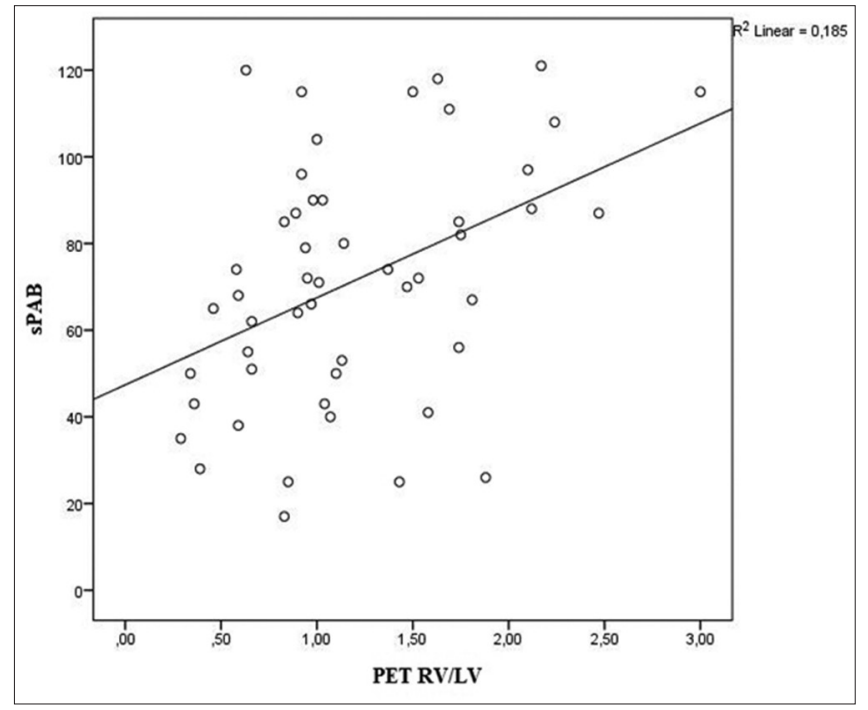

Figure 2: Relationship between 6-MWT and RV and LV FDG PET uptake 


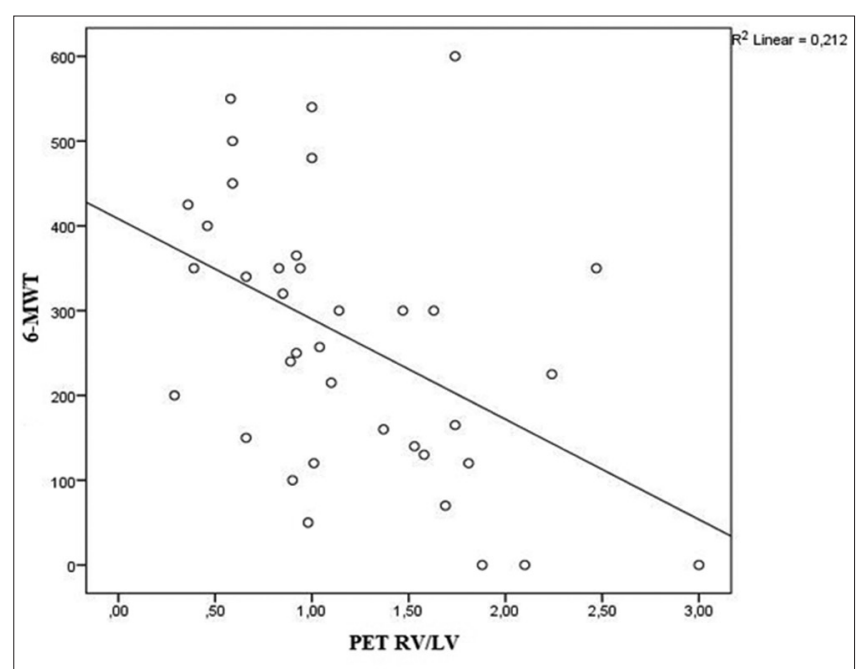

Figure 3: Correlation between echocardiographic parameters and FDGPET uptake

was a significant correlation between RHC parameters diastolic PAP, diastolic pressure gradient and PVR, and FDG-PET uptake [Figure 4]. However, there were no significant correlations between other RHC parameters as cardiac output, cardiac index, and pulmonary capillary wedge pressure (PCWP) [Table 4].

\section{Discussion}

Noninvasive evaluation of RV functions, which have a crucial role in morbidity and mortality in $\mathrm{PH}$, is essential for diagnosis,

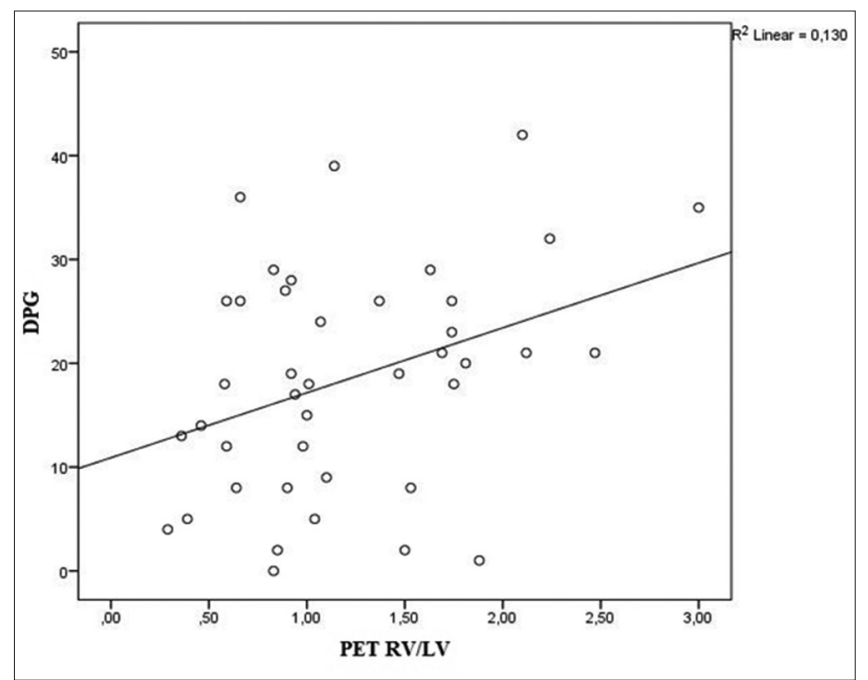

Figure 4: Relationship between diastolic transpulmonary gradient and FDG-PET uptake

risk classification, and determining the therapeutic strategy prognosis of $\mathrm{PH}$ patients. $\mathrm{PH}$ causes remodeling in $\mathrm{RV}$ due to chronic pressure burden. The critical role of RV remodeling in prediction makes RV functions important in studies of PAH. However, the reviews on this topic remained inadequate to be reflected in the clinical approach to the disease. In our research, RV functions were evaluated in patients with CTEPH that planned to have PEA, by a diagnostic modality of FDG-PET/ $\mathrm{CT}$; and its associations with 6-MWD distance, Qanadli score, pro-BNP, other blood parameters, RHC parameters,

Table 3: Right ventricle FDG-PET uptake of study population

\begin{tabular}{|c|c|c|c|}
\hline & $\mathrm{RV} / \mathrm{LV} \leq 1(n=27)$ & $\mathrm{RV} / \mathrm{LV}>1(n=26)$ & $P$ \\
\hline 6-MWT (meters) & $337 \pm 143.1$ & $191 \pm 148.8$ & 0.005 \\
\hline Qanadli score & $21.1 \pm 6.8$ & $24.9 \pm 8.9$ & 0.138 \\
\hline $\operatorname{ProBNP}(\mathrm{pg} / \mathrm{ml})$ & $448(15-6791)$ & $1799.5(12-17020)$ & 0.041 \\
\hline Mean Pulmonary Artery Pressure (mmHg) & $45.7 \pm 17.0$ & $50.4 \pm 14.2$ & 0.298 \\
\hline PCWP $(\mathrm{mmHg})$ & $11.1 \pm 4.5$ & $10.6 \pm 4.0$ & 0.699 \\
\hline Cardiac Output ( 1t/dk) & $4.4 \pm 1.5$ & $3.9 \pm 1.8$ & 0.296 \\
\hline Cardiac Index $\left(\mathrm{lt} / \mathrm{dk} / \mathrm{m}^{2}\right)$ & $2.4 \pm 0.7$ & $2.1 \pm 1.1$ & 0.357 \\
\hline Transpulmonary gradient (mmHg) & $35.0 \pm 17.6$ & $40.7 \pm 13.2$ & 0.250 \\
\hline Diastolic pulmonary gradient $(\mathrm{mmHg})$ & $16.0 \pm 10.1$ & $20.9 \pm 11.3$ & 0.147 \\
\hline PVR (woods) & $9.2 \pm 6.0$ & $11.6 \pm 9.9$ & 0.170 \\
\hline SVR (woods) & $21.6 \pm 8.2$ & $22.1 \pm 9.9$ & 0.858 \\
\hline TAPSE (mm) & $14.8 \pm 4.6$ & $14.0 \pm 4.1$ & 0.552 \\
\hline MPI (mm) & $0.65 \pm 0.09$ & $0.62 \pm 0.22$ & 0.684 \\
\hline RVS (mm) & $11.3 \pm 3.1$ & $10.1 \pm 3.0$ & 0.203 \\
\hline RA area $\left(\mathrm{cm}^{2}\right)$ & $21.8 \pm 7.4$ & $26.0 \pm 7.6$ & 0.06 \\
\hline LA area $\left(\mathrm{cm}^{2}\right)$ & $16.6 \pm 3.9$ & $15.7 \pm 4.4$ & 0.487 \\
\hline RVEDD (mm) & $38.6 \pm 5.2$ & $41.6 \pm 7.6$ & 0.142 \\
\hline LVEDD (mm) & $45.3 \pm 6.0$ & $41.8 \pm 5.6$ & 0.039 \\
\hline LVESD (mm) & $28.4 \pm 4.3$ & $27.1 \pm 5.5$ & 0.364 \\
\hline sPAP (mmHg) & $66.2 \pm 27.8$ & $76.4 \pm 28.7$ & 0.211 \\
\hline
\end{tabular}




\begin{tabular}{|c|c|c|}
\hline & $r$ & $P$ \\
\hline Age (range) - year & 0.200 & 0.156 \\
\hline 6-MWT (meters) & -0.460 & 0.004 \\
\hline Qanadli score & 0.358 & 0.023 \\
\hline D-dimer (mg/L) & 0.585 & 0.002 \\
\hline ProBNP (pg/ml) & 0.397 & 0.018 \\
\hline TAPSE (mm) & -0.215 & 0.138 \\
\hline MPI (mm) & -0.241 & 0.123 \\
\hline RVS (mm) & -0.270 & 0.072 \\
\hline RA area $\left(\mathrm{cm}^{2}\right)$ & 0.393 & 0.005 \\
\hline LA area $\left(\mathrm{cm}^{2}\right)$ & -0.218 & 0.142 \\
\hline RVEDD (mm) & 0.222 & 0.148 \\
\hline LVEDD (mm) & -0.467 & 0.001 \\
\hline LVESD (mm) & -0.337 & 0.018 \\
\hline $\mathrm{sPAP}(\mathrm{mmHg})$ & 0.431 & 0.002 \\
\hline Systolic Pulmonary Artery Pressure (mmHg) & 0.309 & 0.050 \\
\hline Mean Pulmonary Artery Pressure (mmHg) & 0.244 & 0.092 \\
\hline Diastolic Pulmonary Artery Pressure (mmHg) & 0.334 & 0.033 \\
\hline PCWP (mmHg) & -0.101 & 0.523 \\
\hline Cardiac Output ( 1t/dk ) & -0.166 & 0.249 \\
\hline Cardiac Index $\left(1 \mathrm{t} / \mathrm{dk} / \mathrm{m}^{2}\right)$ & -0.129 & 0.376 \\
\hline Transpulmonary gradient (mmHg) & 0.319 & 0.042 \\
\hline Diastolic pulmonary gradient (mmHg) & 0.360 & 0.021 \\
\hline PVR (woods) & 0.297 & 0.038 \\
\hline SVR (woods) & 0.107 & 0.469 \\
\hline
\end{tabular}

RVEDD: Right ventricle end-diastolic diameter LVEDD: Left ventricle end-diastolic diameter; LVESD: left ventricle end systolic diameter; EF: Ejection fraction; RA: Right atrium area; LA: Left atrium area; sPAP: Systolic pulmonary arterial pressure; TAPSE: tricuspid annular plane systolic excursion; RVS: tricuspid annulus tissue Doppler systolic velocity; LV: Left ventricle; PVR: Pulmonary vascular resistance; SVR: Systemic vascular resistance

and echocardiography parameters were evaluated. Lundgrin et al. ${ }^{[10]}$ determined in their study on PAH patients that there was an increase in the synthesis of hypoxia-inducible factor- $1 \alpha$ in pulmonary vascular bed, and this factor caused a shift to nonoxidative glycolysis by affecting mitochondrial metabolism in RV myocytes, which partly explained the ischemic biochemical mechanism that developed in RV. Fang et al. ${ }^{[11]}$ conducted a study on 25 patients with $\mathrm{PH}$ and 43 patients with $\mathrm{PAH}$ associated with congenital heart disease, and at the end of 2 years of follow-up, they compared the RV/LV FDG-SPECT uptake rates and patients' prognosis and reported that patients with higher RV FDG uptake had a worse prognosis. Our study is conducted on patients in a subcategory of $\mathrm{PH}$, the CTEPH patients, and RV/LV FDG-PET uptake ratios were compared with 6-MWD - which is an important parameter that related with functional capacity and prognosis of patients, echo parameters - which are frequently used, noninvasive, and easy-to-perform determinants of RV functions, RHC parameters - which are considered as the gold standard for hemodynamic measurements in PH, and Qanadli score - which determines the pulmonary artery thrombus burden. Based on significant associations with frequently used parameters that assess RV functions, FDG-PET uptake ratio was shown to be a valuable method that can be used for diagnosis and follow-up of patients with CTEPH. However, our theory must be supported with an extensive trial and proved by RHC.

Kepez et al ${ }^{[12]}$ reported in their study on CTEPH patients that decreased RV functions determined by echocardiography were correlated with 6-MWT, which is an indicator of functional capacity. In our research, 6-MWD distances were found to be lower in Group 2 patients that have increased RV FDG-PET uptake, when compared with Group 1 patients $(P=0.005)$. Farber et al. ${ }^{[13]}$ reported in their study that 6-MWD had prognostic importance in $\mathrm{PH}$ patients, and temporally decreased 6-MWD was related to poor prognosis. In this study, 1-year survival was reduced to $68.4 \%$ in patients with $6-\mathrm{MWD}<165$ m. ${ }^{[1]}$ The higher RV/LV FDG-PET uptake in patients with 6 -MWD $<165 \mathrm{~m}$ in our study showed the prognostic value of RV/LV FDG-PET uptake ratio $(P=0.008)$. Despite the primary pathology was on the pulmonary vascular bed as expected, the main reason for symptoms and decreased effort capacity in the disease is increased ischemia due to pressure load, and reduced RV functions. RV FDG-PET uptake can be an essential alternative diagnostic method and prognostic modality in cases that 6-MWD cannot be performed due to reduced effort capacity, advanced age, orthopedic barriers, or comorbid diseases. Functions of RV decrease progressively in patients with high thrombus burden due to theoretically higher PAP and consequently higher pressure load on RV. Qanadli score, which determines acute thrombus burden in pulmonary artery bed, was used for the first time in this study to assess chronic thrombus burden. ${ }^{[7]}$ Qanadli score was found to be associated with RV FDG-PET uptake $(P=0.023)$. Higher PVR develops when thrombus amount increases in the pulmonary vascular bed. Increased PVR causes increased pressure load on RV, adverse remodeling in RV, and decreased RV functions. RV FDG-PET uptake is a valuable diagnostic modality candidate for indirectly showing thrombus burden in the pulmonary vascular bed, and predicting thrombus amount that can be removed by PEA from vascular bed during the planning of CTEPH treatment and subsequently postoperative clinical improvement. Pro-BNP, which is synthesized in myocardial cells that exposed to volume and pressure load, and which is a frequently used blood parameter in left heart failure, was found to be correlated with RV FDG-PET uptake as expected due to the high-pressure gradient. This shows that Pro-BNP is an essential parameter in clinical follow-up and treatment of CTEPH, which provides vital information about disease severity and can be used in the clinical monitoring of $\mathrm{PH}$ patients with these properties.

D-dimer is used in the diagnostic algorithm of acute pulmonary thromboembolism as a fibrin degradation product and more preferred in diagnostic exclusion of acute pulmonary thromboembolism. However, previous studies showed that it has no prognostic value in CTEPH patients, despite being related to disease prognosis in PH patients. ${ }^{[14,15]}$ In our study, we found that serum D-dimer levels were associated with RV/ LV FDG-PET uptake ratio $(P=0.002)$. The high levels of 
D-dimer in patients with increased RV/LV FDG-PET uptake suggest that thromboembolism cycle and in situ thrombus cycle continues in particular in distal arterial bed. These high-risk patients with ongoing in situ thrombus cycle may have a higher risk regarding residual $\mathrm{PH}$ after PEA, particularly following distal arteriopathy.

In our study, we have evaluated the associations between echo parameters that assessed RV functions and RV FDG-PET uptake. Accordingly, FDP uptake was significant in patients with elevated systolic PAP and right atrium area. Recent studies about RV functions also reported similar findings, and most frequently used and most important parameter among those, the TAPSE, was accepted to show RV functions. Li et al. ${ }^{[16]}$ evaluated RV functions by three-dimensional-real-time echocardiography and reported that RV ejection fraction and RV fractional area change are correlated with RV tissue Doppler systolic wave and myocardial performance index, but not with TAPSE. The authors suggested that oscillation of RV apex significantly decreases in a minimal volume or pressure load due to its elastic structure, and this yields TAPSE to be measured less that it is. The limited abilities of echo parameters that are frequently used in the evaluation of RV functions, and their changeability according to operator increases the importance of a more objective evaluation method, the RV FDG-PET.

Diagnosis and treatment of $\mathrm{PH}$ are primarily based on hemodynamic parameters of RHC. According to the European Society of Cardiology (ESC) $2015 \mathrm{PH}$ diagnosis and treatment guidelines, $\mathrm{mPAP} \geq 25 \mathrm{mmHg}$ and $\mathrm{PCWP} \leq 15 \mathrm{mmHg}$ for precapillary $\mathrm{PH}$ and $\mathrm{mPAP} \geq 25 \mathrm{mmHg}$ and $\mathrm{PCWP}>15 \mathrm{mmHg}$ values remained, the transpulmonary gradient was removed, and diastolic pressure gradient and PVR were added to criteria for revised PH diagnosis. This revision was made due to systolic and mPAP were affected by PCWP during the cardiac cycle. ${ }^{[15]}$ A significant correlation between RV FDG-PET uptake and the diastolic PAP, PVR, transpulmonary gradient, and diastolic pressure gradient, which are all hemodynamic parameters of RHC that is the gold standard in diagnosis, treatment, and follow-up of PAH patients. However, there were no significant correlations between systolic and mean PAP, cardiac output and cardiac index, and RV FDG-PET uptake. As Vachiéry et al. ${ }^{[17]}$ reported in their study that referenced in ESC 2015 guideline, systolic and mean PAP is affected by PCWP during the cardiac cycle. Transpulmonary gradient loses its significance in discrimination of $\mathrm{PH}$ as precapillary or postcapillary. However, diastolic PAP and diastolic pulmonary gradient show precapillary $\mathrm{PH}$, since diastolic PAP is affected by PCWP minimally during the cardiac cycle. But, since mPAP, which is the primary diagnostic criteria of $\mathrm{PH}$, is also indirectly affected from PCWP, a similar revision can be done for patients who are in the grey zone with mPAP 21-24 mmHg and can be subject to misdiagnosis due to doubts about the diagnosis. At this point, utilization of diastolic PAP for PH diagnosis by determining a reference range can be discussed, because diastolic PAP is affected very less than mPAP from PCWP.
Furthermore, since we found that RV FDG-PET uptake is correlated with RHC parameters, which are the gold standard in $\mathrm{PH}$ diagnosis, and which were revised in new ESC PH guidelines; right ventricle FDG-PET/CT can be a diagnostic method that can be used along with RHC for PH diagnosis in the future.

Specific treatment in $\mathrm{PH}$ is applied by targeting pulmonary arterial bed. However, symptoms and prognosis of the disease are determined by right heart failure. Right ventricle-targeted therapies have not been evaluated in detail in patients with PAH and right heart failure. The most promising data are about metabolic modulation among right ventricle-targeted medications. Phase 1 and 2 trials of mitochondrial modulators like dichloroacetate have been completed in patients with metabolic changes in the right heart and pulmonary vascular bed in PH. ${ }^{[18]}$ Today, research is needed about stem cell and gene therapies targeting $\mathrm{RV}$, specifically in $\mathrm{PH}$.

\section{Limitations of the study}

Significant limitations of this study are mainly about the low number of patients, which caused many parameters to have a marginal statistical significance that could not reach to relevance. The follow-up of patients after the operation could not be done, and associations of right ventricle FDG-PET with long-term prognosis could not be evaluated. Furthermore, right ventricle FDG-PET evaluations after surgery could not be performed, and more detailed data about RV functions could not be obtained.

\section{Conclusion}

Our study is particularly important for evaluating associations between right ventricle FDG-PET uptake and clinical characteristics, 6-MWD, Qanadli score, echocardiographic, and RHC parameters of CTEPH patients. Under the light of these data, right ventricle FDG-PET uptake was shown to be an innovative noninvasive method that can be used in diagnosis, treatment strategy, and follow-up of CTEPH patients undergoing PEA.

\section{Financial support and sponsorship}

Nil.

\section{Conflicts of interest}

There are no conflicts of interest.

\section{References}

1. Fedullo PF, Auger WR, Kerr KM, Rubin LJ. Chronic thromboembolic pulmonary hypertension. N Engl J Med 2001;345:1465-72.

2. Benza RL, Miller DP, Gomberg-Maitland M, Frantz RP, Foreman AJ, Coffey CS, et al. Predicting survival in pulmonary arterial hypertension: Insights from the registry to evaluate early and long-term pulmonary arterial hypertension disease management (REVEAL). Circulation 2010;122:164-72.

3. McCann GP, Gan CT, Beek AM, Niessen HW, Vonk Noordegraaf A, van Rossum AC. Extent of MRI delayed enhancement of myocardial mass is related to right ventricular dysfunction in pulmonary artery hypertension. AJR Am J Roentgenol 2007;188:349-55. 
4. Ahmadi A, Ohira H, Mielniczuk LM. FDG PET imaging for identifying pulmonary hypertension and right heart failure. Curr Cardiol Rep 2015; 17:555.

5. Rudski LG, Lai WW, Afilalo J, Hua L, Handschumacher MD, Chandrasekaran $\mathrm{K}$, et al. Guidelines for the echocardiographic assessment of the right heart in adults: A report from the American society of echocardiography endorsed by the European association of echocardiography, a registered branch of the European society of cardiology, and the Canadian society of echocardiography. J Am Soc Echocardiogr 2010;23:685-713.

6. Weisman IM, Zeballos RJ. An integrated approach to the interpretation of cardiopulmonary exercise testing. Clin Chest Med 1994;15:421-45.

7. Qanadli SD, El Hajjam M, Vieillard-Baron A, Joseph T, Mesurolle B, Oliva VL, et al. New CT index to quantify arterial obstruction in pulmonary embolism: Comparison with angiographic index and echocardiography. AJR Am J Roentgenol 2001;176:1415-20.

8. Can MM, Kaymaz C, Tanboga IH, Tokgoz HC, Canpolat N, Turkyilmaz E, et al. Increased right ventricular glucose metabolism in patients with pulmonary arterial hypertension. Clin Nucl Med 2011;36:743-8.

9. Saygin D, Highland KB, Farha S, Park M, Sharp J, Roach EC, et al. Metabolic and functional evaluation of the heart and lungs in pulmonary hypertension by gated 2-[18F]-fluoro-2-deoxy-D-glucose positron emission tomography. Pulm Circ 2017;7:428-38.

10. Lundgrin EL, Park MM, Sharp J, Tang WH, Thomas JD, Asosingh K, et al. Fasting 2-deoxy-2-[18F]-fluoro-D-glucose positron emission tomography to detect metabolic changes in pulmonary arterial hypertension hearts over 1 year. Ann Am Thorac Soc 2013;10:1-9.
11. Fang W, Zhao L, Xiong CM, Ni XH, He ZX, He JG, et al. Comparison of $18 \mathrm{~F}$-FDG uptake by right ventricular myocardium in idiopathic pulmonary arterial hypertension and pulmonary arterial hypertension associated with congenital heart disease. Pulm Circ 2012;2:365-72.

12. Kepez A, Sunbul M, Kivrak T, Eroglu E, Ozben B, Yildizeli B, et al. Evaluation of improvement in exercise capacity after pulmonary endarterectomy in patients with chronic thromboembolic pulmonary hypertension: Correlation with echocardiographic parameters. Thorac Cardiovasc Surg 2014;62:60-5.

13. Farber HW, Miller DP, McGoon MD, Frost AE, Benton WW, Benza RL. Predicting outcomes in pulmonary arterial hypertension based on the 6-minute walk distance. J Heart Lung Transplant 2015;34:362-8.

14. Shitrit D, Bendayan D, Bar-Gil-Shitrit A, Huerta M, Rudensky B, Fink G, et al. Significance of a plasma D-dimer test in patients with primary pulmonary hypertension. Chest 2002;122:1674-8.

15. Arunthari V, Burger CD. Utility of d-dimer in the diagnosis of patients with chronic thromboembolic pulmonary hypertension. Open Respir Med J 2009;3:85-9.

16. Li Y, Wang Y, Zhai Z, Guo X, Yang Y, Lu X. Real-time three-dimensional echocardiography to assess right ventricle function in patients with pulmonary hypertension. PLoS One 2015;10:e129557.

17. Vachiéry JL, Adir Y, Barberà JA, Champion H, Coghlan JG, Cottin V, et al. Pulmonary hypertension due to left heart diseases. J Am Coll Cardiol 2013;62:D100-8.

18. Nagendran J, Michelakis ED. Mitochondrial NOS is upregulated in the hypoxic heart: Implications for the function of the hypertrophied right ventricle. Am J Physiol Heart Circ Physiol 2009;296:H1723-6. 